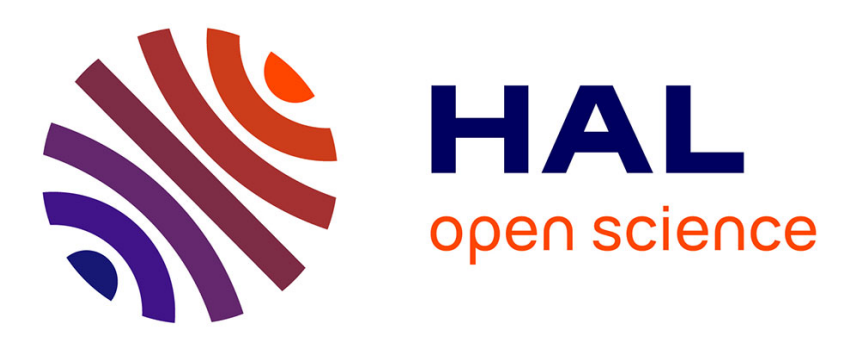

\title{
L'environnement sensible et les ambiances architecturales
}

Jean-François Augoyard

\section{To cite this version:}

Jean-François Augoyard. L'environnement sensible et les ambiances architecturales. Espace Géographique, 1994, 4, pp.302-318. 10.3406/spgeo.1995.3409 . hal-02103999

\section{HAL Id: hal-02103999 \\ https://hal.science/hal-02103999}

Submitted on 11 Jun 2019

HAL is a multi-disciplinary open access archive for the deposit and dissemination of scientific research documents, whether they are published or not. The documents may come from teaching and research institutions in France or abroad, or from public or private research centers.
L'archive ouverte pluridisciplinaire HAL, est destinée au dépôt et à la diffusion de documents scientifiques de niveau recherche, publiés ou non, émanant des établissements d'enseignement et de recherche français ou étrangers, des laboratoires publics ou privés. 


\title{
L'environnement sensible et les ambiances architecturales
}

\author{
Jean-François Augoyard \\ CNRS, URA 1268, Centre de recherche sur l'Espace sonore et l'environnement urbain \\ École d'architecture, 60, av. de Constanfine, 38036 Grenoble Cedex 2
}

\begin{abstract}
RÉSUMÉ- - Au cours de ces vingt dernières années, les recherches sur la ville en tant qu'environnement perceptible ont suivi deux voies complémentaires. D'un côté l'exigence de l'approche in situ pousse les méthodes d'observation du côté de l'analyse modale et appelle l'interdisciplinarité. D'un autre côté, les travaux sur les perceptions de l'environnement construit et la maîtrise des formes sensibles s'interrogent sur le sens des sens, sur les connexions entre norme et invention, usage et technique, modèles de perception et modèles d'action. C'est autour des notions d'environnement sensible et d'ambiances architecturales qu'est en train de se développer aujourd'hui un courant de recherche français doublement instrumenté par la modélisation et par une esthétique des configurations sensibles.
\end{abstract}

AMBIANCES, ARCHITECTURE, ENVIRONNEMENT SENSIBLE, ESTHÉTIQUE, FORME URBAINE, INTERDISCIPLINARITÉ, PERCEPTION IN $S T T$, USAGES QUOTIDIENS
ABSTRACT.- Sensible environment and architectural ambiences.- For the last twenty years, research on the city as a perceptible environment has been going two complementary ways. On the one hand, the necessity for an in situ approach leads the observation methods to the side of modal analysis and appeals to interdisciplinarity. On the other hand, studies on the perceptions of built environment and on the control of the sensible forms ponder over the meaning of the senses, the connections between standards and inventiveness, customs and technique, perception models and action models. Working on the notions of sensible environment and architectural ambiences and using both modelling and aesthetics of the sensible configurations, a French research movement is developing.

AMBIENCES, ARCHITECTURE, CUSTOMS, AESTHETICS, IN SITU PERCEPTION, INTERDISCIPLINARITY, SENSIBLE ENVIRONMENT, URBAN FORM

\section{L'écologie à la rencontre de la ville}

Au cours de l'essor remarquable qu'elles ont connu depuis trois décennies, les sciences de l'environnement n'ont pas manqué d'aborder largement et à leur manière le phénomène urbain. La ville n'engendre-t-elle pas une bonne partie des nuisances repérées et pourchassées? En retour, il était inévitable que la recherche architecturale et la recherche urbaine ne puissent ignorer plus longtemps l'émergence d'une dimension de plus en plus préoccupante de l'espace 
construit, non plus que l'essor concomitant d'outils théoriques et méthodologiques regroupant leurs forces dans une «lutte contre les nuisances» très demandée.

Dans le climat studieux et confidentiel d'une recherche architecturale française longtemps à la recherche de son biotope institutionnel idéal, l'approche environnementale semblait intervenir brusquement par le biais du raccourci techniciste et sur le ton de l'urgence. En effet, à partir des années 1970, la recherche sur la conception de l'espace avait été patiemment constituée comme champ de savoir autour de trois problèmes fondamentaux et en usant librement de réemplois théoriques divers. La question du sens et de l'identité de l'architecture appelait une pensée épistémologique ou sémiologique (1). La question de la spécificité des méthodes descriptives et représentatives de l'objet architectural invitait non seulement à édifier des typomorphologies, mais aussi à chercher du côté de la modélisation (2). Enfin, la question de l'intelligibilité du corpus des références architecturales appelait une démarche historiciste au long cours.

Or, voici que différentes «crises» écologiques imprévues et concernant directement l'espace construit vont se succéder. On attend de la recherche des solutions rapides: ainsi l'urgence de l'isolation thermique face à la crise énergétique, ainsi l'urgence de l'amélioration de I'isolation acoustique face à une demande sociale pressante. Allait-on voir se constituer une recherche hypertélique? Devait-on aussi redouter qu'un écologisme systématique conforté autant par le fléchage des crédits que par le flou des notions - toute situation architecturale et urbaine étant un environnement au sens large - n'investisse et ne gangrène les théories et les méthodes de la recherche sur l'espace construit?

Vingt années de cheminement entre recherche environnementale et recherche sur l'espace construit nous montrent que rien n'a été aussi simple. L'espace

(1) La première représentée d'emblée par Philippe Boudon.

(2) La recherche sur la modélisation architecturale est développée depuis le début des années 1970 par deux laboratoires GAMSAU (École d'architecole d'architecture de Nantes) et le GAMSAU (École d'architecture de Marseille). construit et habité n'est pas un «terrain» quelconque. Sa complexité réserve bien des surprises à l'écologue ou à l'environnementaliste trop pressé. À l'inverse une recherche sur l'environnement architectural ne s'arrêtant pas au discours et au représenté mais soigneusement attentive à la nature sensible et physique des objets observés apporte de nouveaux accès à la connaissance globale du phénomène urbain.

$\grave{A}$ partir de ma participation à ce cheminement interdisciplinaire, je voudrais indiquer quelle relation dialectique positive est née de la rencontre entre les sciences de l'environnement et la recherche urbaine. En quoi l'espace urbain construit a-t-il changé quelque chose dans l'attitude scientifique qui le prenait pour nouvel objet d'étude? Comment la recherche sur l'environnement sensible apporte-telle actuellement du nouveau à la recherche sur l'architecture et la ville?

\section{Le paradigme de la recherche sur le bruit}

Les sciences de l'environnement ont trouvé dans le milieu urbain un condensé remarquable des nuisances existantes: pollutions de l'air et de l'eau, bruit, inconfort lumineux, multinuisances, multi-expositions. C'est pourtant de ce milieu abordé comme terrain d'observation que sont nés les plus grands obstacles épistémologiques. Au regard de l'observateur attentif qui sort du laboratoire pour aller sur le site, la ville apparaît comme un tissu serré de variables contextuelles très interdépendantes et qui résistent aux réductions familières à la méthode expérimentale. En guise de brève illustration de cette rencontre problématique entre une méthode issue des sciences physiques et un objet complexe interdisciplinaire comme la ville, il peut être éclairant de relever les grands traits de l'histoire des programmes de recherche nationaux sur le bruit entre
1975 et 1990 .

Au début des années 1970, la recherche en acoustique et psycho-acoustique jusque-là essentiellement orientée sur la physique fondamentale, l'audiologie, la phonologie, l'acoustique musicale et l'acoustique des salles d'écoute a été sollicitée sur le thème de l'environnement urbain. Les problèmes de nuisance du bruit routier et des bruits au travail commençaient à émerger dans la demande 
sociale. Les programmes de recherche nationale pilotés par le ministère de l'Environnement vont connaître trois périodes entre 1970 et 1990.

Au cours de la première étape fortement polarisée par une urgence sanitaire, on a d'abord cherché à caractériser les effets auditifs et extra-auditifs des nuisance sonores, à les normer (seuils de tolérance, échelles de gêne), et à tester des dispositifs de protection. Au centre de ces travaux, un postulat directement inspiré de la démarche in vitro: il faut distinguer l'action sur les causes et l'action sur les effets. Les remèdes face aux situations de nuisance seront donc soit la modification du bruit à la source, soit l'isolation phonique des individus victimes du bruit. On attend, par ailleurs, que les normes technico-juridiques créées en abondance à cette époque modifient de façon décisive les comportements et les nuisances (3). Pourtant, l'observation au jour le jour et l'examen des jurisprudences montrent que les réglementations sont parfois loin du vécu de la nuisance el que l'application des normes n'est pas nécessairement satisfaisante pour le plaignant (4).

Dans une seconde étape, la recherche va essayer de mieux comprendre les facteurs proprement «humains». On attend beaucoup de l'affinement de la notion de «gêne» abordée à l'interface du physiologique et du psychologique (5). Mais les résultats seront décevants (6). Les moyennes des réactions sont trop imprécises et les variables les plus éclairantes vont souvent se nicher à l'intérieur de l'histoire du sujet. En même temps, les premiers travaux véritablement interdisciplinaires (7) commencent à montrer que le statut

(3) Les travaux de cette époque sont effectués en presque totalité par les sciences pour l'ingénieur et la recherche médicale.

(4) Les causes en sont diverses: émergence croissante d'une sensibilité au bruit, transfert de la quérulence sur un autre objet, victimisation directe, socialisation du conflit... (cf. P. Amphoux et al., Le Bruit, la plainte et le voisin. Grenoble: CRESSON, $1988,2 \mathrm{t}$.

(5) Travaux du CEP (M. Perianez, F. Desbons), du CSTB (D. Aubree).

(6) Cf. C. Levy-Leboyer, dir. Douze ans de recherche sur la gêne due au bruit. Paris: IRAP/SRETIE, 1987.

(7) Cf. les travaux du Centre de recherche sur l'espace sonore et l'environnement urbain (CRESSON, URA CNRS, École d'architecture de Grenoble) entre 1980 et 1986. des normes est multiple et complexe, que le contexte architectural et urbain n'est pas indifférent dans les problèmes de bruit et qu'enfin la nuisance sonore est un cas remarquable à étudier au sein d'une anthropologie plus générale de l'environnement sonore. Positifs ou négatifs, les bruits sont aussi des instruments des relations collectives. Deux conséquences en découlent: les normes sont à relativiser en fonction des conditions expérimentales qui les fondent et, par ailleurs, l'isolation phonique ne serait plus la seule solution curative à recommander dans tous les cas.

La troisième étape, à partir de 1983, va cuvrer plus systématiquement sur les effets du bruit in situ. Le contexte spatial et social du milieu urbain devient alors un objet d'investigation central. Les travaux explorent les dimensions humaines oubliées: la psychologie des faiseurs de bruit, la fonction des représentations autour du bruit (le silence, par exemple), le jeu des interdépendances entre les acteurs de plainte pour bruit de voisinage, la gestion des problèmes de bruit par le pouvoir local et les acteurs sociaux locaux. Fondé sur les travaux précédents, un programme lancé en 1990 est intitulé: «Gestion de l'environnement sonore urbain». Il remet en perspective les travaux plus monothématiques (bruits de transports, de travail, d'équipements) en valorisant les relations complexes entre les caractéristiques physiques et sociales du milieu urbain. Autre inflexion notable: dépassant les seules finalités normatives et défensives, ce programme recherche des outils pour aider la planification et la conception des villes.

De façon complémentaire, d'autres instances de programmation de recherche plus axées sur l'objet urbain, en particulier celles du ministère de l'Équipement, de l'Urbanisme et du Logement, interrogent la composante environnementale ou sensible (8). Pour résumer, on pourrait dire que la tendance actuellement partagée est d'étudier les dimensions contextuelles et de chercher à mieux utiliser les variables qualitatives.

(8) Ce mouvement d'interrogation des composantes environnementales émane en particulier du Plan Urbain et plus récemment du programme interdisciplinaire sur la ville (Pir-Ville $d u$ CNRS). 


\section{L'épreuve du in situ}

Parmi les composantes perceptibles (9) de l'environnement architectural et urbain qui sont objets de recherche - c'està-dire la lumière, le son, la matière tactile, l'air qualifié par la température, la vitesse et l'odeur -, le bruit présente certainement l'exemple le plus développé et le plus complexe d'un retour de l'objet au contexte. En voici au moins quatre raisons: la difficulté à trouver des normes et des dispositifs physiques pleinement satisfaisants dans les situations sonores courantes (10), l'extraordinaire charge de connotations psychologiques et sociologiques qui viennent peser sur les perceptions sonores, la perdurance d'une demande sociale d'autant plus forte qu'elle reste insatisfaite des solutions techniques et juridiques proposées, enfin, le profil singulier des organismes et des responsables de la recherche dans ce secteur.

Il faut bien remarquer que les autres composantes de l'ambiance urbaine n'ont pas connu un effort de développement aussi soutenu, non plus qu'une interdisciplinarité aussi ouverte, en particulier du côté des sciences humaines. Ainsi, les rares et intéressants travaux sur la perception individuelle et sociale des phénomènes thermiques ont été abandonnés depuis quelques années, un seul laboratoire de recherche architecturale développant actuellement la prise en compte des valeurs d'usage entre la composante technique et celle du projet d'architecture (11). Ainsi, les recherches sur la perception de la lumière en site urbain ne

(9) On voit qu'il ne s'agit donc pas de la totalité des composantes de l'environnement de la ville, mais de celles dont la qualité est appréciable par les sens avec une précision suffisante pour 2) être support les puissent 1) être conscientes et verbalisables; construites; 3) être intégrées dans l'inétiques et axiologiques social; 4) être rapportées grées dans l'instrumentation du lien gie spatiale (cf. infra. L' au moins intuitivement, à la morpholo-

(10) (cr. infra: L'Environnement sensible)

(10) Hormis les situations acoustiques spécialisées et monofonctionnelles: bruit routier, écoute de spectacles, écoute profes-
sionnelle, etc.

(11) Les travaux de sociologie de l'usage dans le domaine thermique ont été développés au CSTB par Philippe Dard. Le laboratoire de recherche architecturale qui, tout en développant la modélisation de la thermique appliquée au projet d'architecture, de l'École d'architecture d'usage est le CERMA (URA CNRS) de l'École d'architecture de Nantes dirigé par J.P. Peneau. réussissent pas encore à s'affranchir de l'ergonomie visuelle routière ou fonctionnelle. On s'en convaincra en lisant les plaquettes de recommandation d'éclairement diffusées dans les services techniques municipaux. Malgré de surabondantes études sur la vision, on ne sait pas grand chose sur l'ensemble des composantes de l'attitude visuelle d'un piéton, la nuit (12). Enfin, sur les autres sens qui nous informent sur l'environnement urbain, malgré quelques travaux isolés (13), aucun champ de savoir interdisciplinaire n'existe encore (14). L'exemple du bruit montre donc de la façon la plus développée ce qui peut arriver aux sciences de l'environnement quand elles abordent l'objet urbain.

Que se passe-t-il lorsqu'un chercheur sort du laboratoire pour observer in situ la complexité des phénomènes d'environnement urbain? Plus riche est l'information et plus tangible la saisie des interdépendances observables. En même temps, certaines limites de la méthode du in vitro que l'on espérait réemployer dans le site apparaissent clairement.

Quelles sont ces limites? Nous pouvons en citer au moins cinq parmi les plus remarquables: 1) délocalisation des problèmes à traiter, en faveur de l'universalité de l'énoncé; 2) orientation des finalités vers la production de normes et de dispositifs techniques universels dont on attend une remédiation efficace; 3) limitation de I'observation de l'humain soit à l'appareil neurologique récepteur, soit au sujet type; 4) réemploi du modèle d'intelligibilité des

(12) Un article fondateur avait été rédigé voici une dizaine d'années par le regretté Abraham Moles: «Des fonctions de la lumière dans la ville». (Communication aux Journées natio10-25. Cf, aussiere, Paris, septembre 1980), in LUX n 111, p. Les Facteurs lumineux d'un séminaire: J.-F. Augoyard dir., CRESSON, 1990.

(13) Ainsi, les travaux bien connus d'Alain Corbin, ou encore ceux des historiens du paysage (K. Pomian, J.-D. Hundt,
A. Roger, Y. Nakamura).

(14) Le développement de la recherche sur les sens paraît être extrêmement lié à des impératifs de maîtrise technique ou scientifique d'une situation d'intérêt immédiat: ainsi à côté de quelques rares recherches générales sur les odeurs, on trouvera une énorme littérature grise sur la dimension olfactive du comdes souches de rats SPF : optimiser la sélection et la maîtrise laboratoires de biologie. 
sciences physiques pour traiter l'ensemble de la chaîne des effets causés par le signal (15); 5) enfin, choix d'une attitude axiologique défensive que l'on peut résumer par le double emboîtement suivant: l'environnement est à étudier quand il devient nuisance; cette nuisance paraît d'autant plus évidente qu'elle a des effets mesurables sur la santé (16).

Une analyse historique et typologique des comités scientifiques ayant piloté la recherche sur l'environnement montrerait certainement un clivage remarquable, peu déterminé par les origines disciplinaires, entre les scientifiques travaillant in vitro et ceux qui pour diverses raisons - recherche appliquée de faisabilité, validation pratique des outils et résultats, enquêtes sur des populations situées -, ont dû fréquenter assidûment le terrain urbain. La découverte de la complexité structurelle de la ville entraîne immanquablement une lente évolution des manières d'envisager l'environnement.

Ce qui est alors remis en cause, c'est moins la validité des modèles scientifiques majeurs qui ont fait leurs preuves que le postulat d'une intangible hiérarchie d'excellence entre modèles. Découverte que l'environnement construit est plus qu'une collection de signaux mesurables et de dispositifs techniques. Découverte des causes adjacentes par lesquelles une législation n'est pas appliquée ou non respectée, un dispositif de défense contre les nuisances est détourné ou perverti. Découverte qu'une nuisance physique peut avoir des effets sociaux positifs (associations, solidarités sociales, incitation à la démocratie directe). Découverte, donc, que la ville

(15) D'où les approximatives torsions infligées au qualitatif qu'il faut bien réintroduire dans l'analyse complexe des réactions humaines. On se référera à l'histoire du décibel qui ne réussit pas à être une vraie mesure physique, ou encore aux notions hybrides de sonie, de bruyance qui représentent les traces d'une subjectivité appelée à la rescousse dans la dernière partie des traités d'acoustique appliquée. Sur la critique épistémologique des réemplois scientifiques improbables, on peut lire avec profit deux ouvrages très récents: I. Stengers, L'Invention des sciences modernes. Paris: La Découverte, 1993; G. Chatelet, Les Enjeux du mobile, Mathématique, physique, philosophie. Paris: Le Seuil, 1993.

(16) D'où une tendance à donner priorité de droit aux recherches médicales. observée in situ exige un pluralisme dans les hypothèses, dans les méthodes et dans les développements théoriques.

Pour résumer l'évolution remarquable que connâ̂t la recherche environnementale à partir du moment où elle s'intéresse au milieu urbain, on pourrait donc dire que l'objet observé fini par modifier l'observateur, c'est-à-dire ses techniques d'investigation et sa manière de penser l'observable.

\section{Les raisons écologiques de l'intelligibilité de l'urbain}

Par les résistances du terrain à la logique du in vitro familière au secteur des sciences de l'environnement à dominante physique, l'urbain fait donc valoir un certain nombre de raisons qui tiennent à la très forte récurrence du contexte sur les phénomènes observables er, par ailleurs, à des modèles d'intelligibilité atypiques.

\section{Confextualifé récurrente}

Pourquoi certaines conditions d'apparitions des phénomènes observables que la physique expérimentale distinguerait comme étant du contexte contingent sont-elles parties intégrantes de l'objet observé? C'est précisément qu'in situ on ne trouve pas de phénomène pur. Rappelons d'abord que, dans l'environnement urbain, tout signal physique est instrumenté par d'autres éléments physiques. Le son situé traverse un espace de propagation qui lui donne une certaine qualité hic et nunc; par exemple: temps de réverbération et timbrage pour le son, réflexion particulière, modification de température, de couleur, organisation des ombres pour la lumière, turbulences particulières des flux de l'air autour de certaines configurations architecturales, volatilité variable des odeurs en fonction de la vitesse du vent. Le signal physiquement isolable a posteriori n'existe qu'à travers cette incorporation spatio-temporelle entièrement dépendante des qualités morphologiques et matérielles du lieu. D'ailleurs, parmi ces conditions essentielles, toutes ne sont pas sujettes à mesure; il n'y a pas d'équivalent du décibel, par exemple, pour les vibrations ou les odeurs. Aussi, les praticiens de l'environnement urbain poussent-ils actuellement la recherche à trouver des modélisations multicritères qui intégreraient des variables qualitatives. 
La qualitativité de l'environnement urbain découle d'abord, par défaut, de la part du non mesurable dans une situation urbaine. Elle tient encore et plus généralement au fait qu'in situ tout signal physique n'a de sens que s'il est perceptible. Pas d'en-soi du signal. La variabilité n'est pas accidentelle. Le même son, la même lumière peuvent parfaitement être connotés de sens et de valeurs différents, voire antithétiques en fonction des sujets, des groupes sociaux, des moments de perception. Ils ne sont donc jamais exactement les mêmes, ni par ce qui est filtré du signal, ni par les connotations qui les chargent. Au gré de cette variance, une identique composition de signaux (le passage sonore d'un train, un panneau publicitaire lumineux et animé) sera susceptible d'être aussi bien support de pratique utilitaire qu'indice normatif, qu'objet d'une dynamique réactionnelle, qu'objet esthétique. Du côté de la perception, la prégnance des facteurs individuels, sociaux, culturels, économiques projette la contextualité au cœur du phénomène.

Dira-t-on que tout sombre alors dans la confusion? Il est vrai que la ville, ce compendium du monde où sont rassemblés, coexistent et prolifèrent les objets, les pratiques et les représentations les plus variés, est particulièrement exposée aux rêveries qui font coïncider les contraires avec plus ou moins de bonheur, aux exégèses dilettantes qui mélangent allégrement les genres, aux essais tout aussi amnésiques qu'insouciants de rigueur dont les exemples ne manquent pas, aujourd'hui comme hier.

Pourtant, la complexité n'implique pas nécessairement le chaos ou l'équivalence généralisée. Et l'interdépendance n'interdit pas la distinction formelle entre les termes en présence. Les recherches sur l'environnement architectural et urbain qui ont abouti à quelque résultat montrent que la coordination réussie entre approches différentes est basée sur la distinction des compétences et sur une autonomie minimale entre genres de connaissance. Sous ces conditions, la prise en compte du qualitatif ne signifie pas l'exclusion du quantitatif. Les chercheurs pratiquant le terrain savent combien les deux dimensions peuvent s'enrichir mutuellement et que le phénomène remarquable apparaîtra aussi bien au détour d'une observation ethnographique, qu'au cours du dépouillement de telle mesure. D'autre part, plus la situation est complexe et plus la patience dans l'investigation, plus la prudence dans les conclusions sont requises pour qui souhaite retrouver avec rigueur le fil des relations et des interdépendances. Et plus le regard sur l'observable s'émancipe de l'empreinte exclusive des sciences physiques.

\section{Évolution des modèles d'intelligibilité}

Une approche écologique de l'espace construit visant à saisir les phénomènes perceptibles situés suppose, en fait, une mutation des modèles d'intelligibilité. Certains caractères de ce changement en cours peuvent être aujourd'hui identifiés. Notons d'abord que la nécessaire prise en compte de la dimension anthropologique engage une double confrontation. En face du sujet type de laboratoire, cible des stimuli, se dessine le citadin concret, pénétré de son histoire individuelle, de son imaginaire, des interactions singulières qu'il entretient avec son réseau de sociabilité, mais aussi acteur de son environnement, producteur de signaux, voire de nuisances. Par ailleurs, en face des populations statistiques, vivent groupes et communautés reliés à l'environnement par la médiation d'une interaction indissoluble entre le sensible et le social qui s'instrumentent mutuellement. Les chaînes causales entre les différentes composantes du phénomène étudié ne sont donc plus univoques et linéaires. Il y a, d'une part, convergence entre différents systèmes de causes et de conditions pour définir la nature et l'existence du phénomène localisé et, d'autre part, interdépendance des facteurs en action.

Le second caractère remarquaủe commence à faire partie des évidences, sinon des pratiques effectives: c'est l'interdisciplinarité. Cette attitude faisant interagir diverses compétences dans une parité de droit ne va pas de soi. La recherche sur les nuisances a souvent pratiqué une pluridisciplinarité du dernier recours. Autrement dit, non seulement l'interdisciplinarité, entendue comme l'échange et la subsomption de chaque spécialité sous des concepts communs et des méthodes intégratives, n'a pas eu lieu, mais l'ouverture du modèle physique sur d'autres paradigmes de compréhension se produit seulement lorsque la démarche bute sur l'inconnaissable. La «boîte noire» serait alors qualifiée par d'hypothétiques variables sociales et culturelles qui viendraient brouiller la clarté du rapport causal entre le signal physique et ses effets physiologiques. Dans cet état d'esprit, les indices permettant de repérer ces variables ne bénéficient évidemment d'aucune autonomie sémantique. Ils renvoient seulement aux dégradations occasionnelles 
des lois causales fondées sur le modèle unilinéaire allant du plus connu, c'est-à-dire du plus quantifiable, au plus incertain. L'histoire de la recherche sur la gêne sonore évoquée plus haut donne un excellent exemple de cette fausse pluridisciplinarité.

Voici bientôt trente ans, Pierre Schaeffer faisait la critique argumentée de l'extrapolation du modèle physique pratiquée sans discernement en musicologie, en psychologie de la musique, mais aussi dans le discours plus commun des musiciens, des pédagogues, des auditeurs éclairés. Pourtant, la construction de l'intelligibilité des phénomènes sonores à partir du signal ne vaut que dans les limites du champ expérimental qui fonde ce type de connaissance (17). D'autres accès à la compréhension de l'écoute en général et, particulièrement, de l'écoute in situ, sont tout aussi légitimes. Tout dépend de l'angle selon lequel le phénomène est observé. On ne peut pas toujours dire: «au début, était le signal».

Ce renversement épistémologique ouvre la porte au pluralisme des modèles d'intellection dont la distinction n'est plus que formelle (du point de vue de tel savoir, de telle discipline) et dont l'exploration d'ensemble aboutit à la définition d'un objet central qui n'est plus le signal mais le phénomène. On peut représenter le développement actuel des connaissances sur l'environnement perceptible par un schéma radial (fig. 1). Un phénomène d'ambiance situé s'incorpore en six modes complémentaires: il est signal physique, forme spatio-temporelle, percept, représentation, élément d'un code ou d'une norme, instrument d'interaction sociale. Chacun des modes requiert des techniques d'investigation, des méthodes d'analyse et des langages spécialisés, non équivalents. Mais ce déploiement d'approches convergentes ne reste-t-il pas simplement pluridisciplinaire, ne cultive-t-il pas le voisinage des savoirs à de seules fins spéculatives?

(17) P. Schaeffer (1966). Traité des objets musicaux. Paris: Le Seuil, Livres I et II.

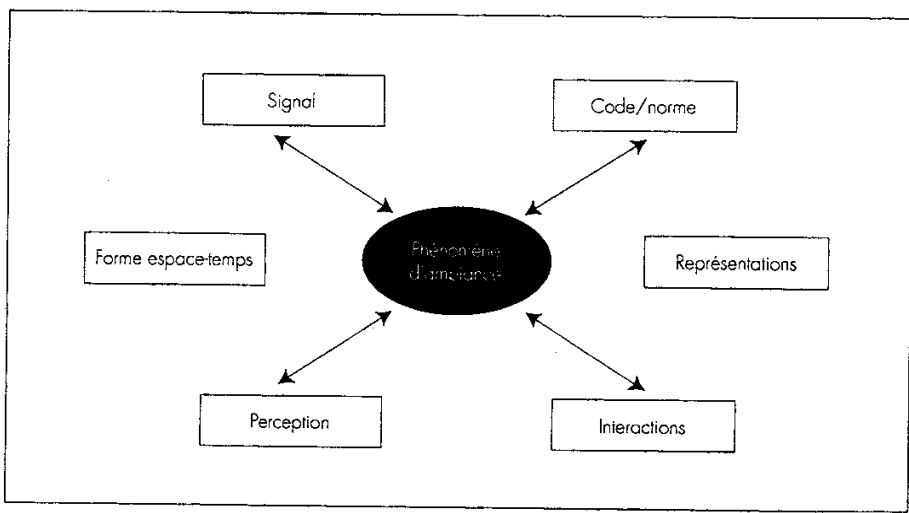

Fig. 1.--Modalités d' un phénomène d'ambiance in situ.

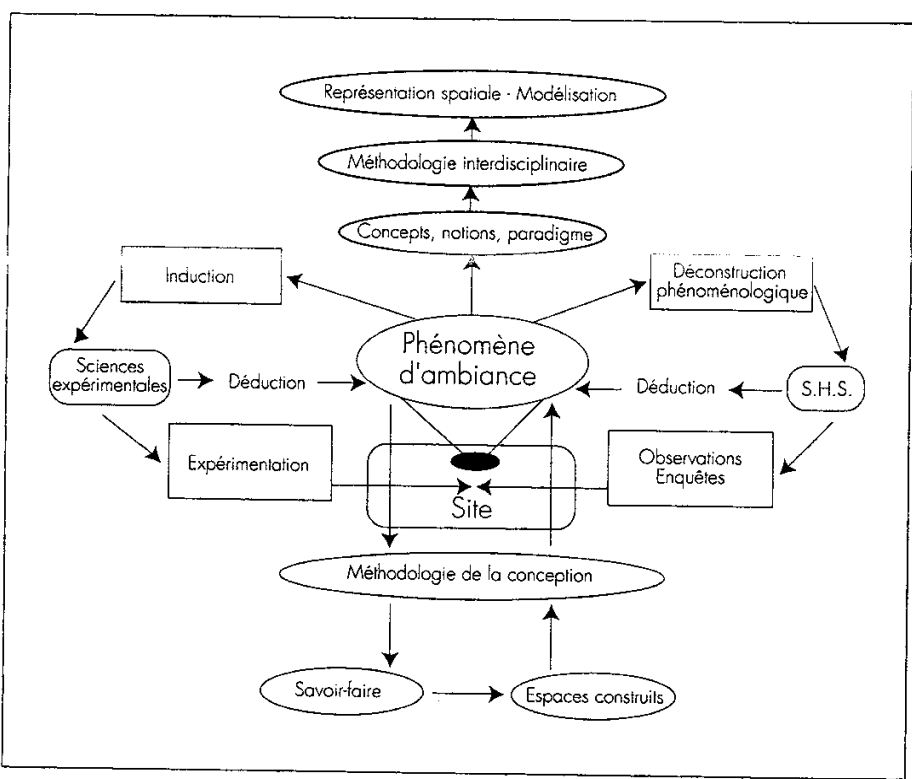

Fig. 2.- Analyse interdisciplinaire

d'un phénomène d'ambiance in situ.

La figure 2 tente de représenter comment la diversité de deux approches, parmi les plus connues et très souvent opposées, trouve une complémentarité opératoire et donc interdisciplinaire. Dans la démarche, la spécificité des paradigmes et plans d'observation ou d'expérimentation est respectée. L'interaction a lieu en cinq points de contacts: sur le site, dans la récolte des résultats, dans la production de concepts transversaux, dans le développement de modèles d'intelligibilité, dans l'application des outils interdisciplinaires.

Sur le site commun, l'interaction est manifeste par l'enrichissement de la liste des objets observables en fonction des émergences électives: telle mesure surprenante appellera tel 
examen morphologique et telle question d'enquête non prévue dans le protocole initial; telle remarque d'un usager invitera à faire ou à diversifier telle mesure.

La comparaison interdisciplinaire des résultats est d'autant plus utile si elle intervient à chaque phase de la recherche et permet ainsi un affinement ou une rectification progressive des hypothèses.

La production de concepts transversaux n'est jamais facile, ni rapide. La première intuition appelle de longues vérifications de l'extension et de la compréhension du terme qui, parfois, n'accède pas au statut de concept. Il est tout aussi long de valider les modalités d'application du concept dans toutes les disciplines concernées et dans tous les genres de situation où il serait applicable (18).

Le développement des modèles d'intelligibilité intégrateurs peut prendre des formes diverses: théorie interdisciplinaire, modélisations procédurales ou déclaratives, vérification critique de modèles importables, conception de démarches méthodologiques types.

Enfin, l'épreuve de l'application des outils interdisciplinaires sur le site est particulièrement idoine dans la méthodologie de la conception architecturale et urbaine. Dans ce domaine, en effet, le lien entre société et recherche est patent. Si le champ des savoirs et savoir-faire urbains et architecturaux est une mine abondante de questions posées à la recherche interdisciplinaire, en retour, la valorisation pratique des outils est à considérer non seulement comme un devoir social mais aussi comme une chance de progresser dans une saisie des phénomènes non seulement attentive à la facticité mais aussi à l'opérativité.

\section{Penser des processus}

Précisément, le troisième trait remarquable de l'évolution actuelle des façons d'approcher l'environnement architectural

(18) À titre d'exemple, la notion d'effet sonore avancée au CRESSON en 1978 pour rendre compte du caractère interdisciplinaire des phénomènes in situ a dû être vérifiée durant sept années. La validation de ses applications et de sa qualité prédictive commencée en 1988 est encore en chantier. et urbain, c'est le passage progressif d'une pensée de l'état à celle de processus. Le secteur des sciences de l'environnement, occupé à l'étude des facteurs physiques urbains (aéraulique, thermique, acoustique, éclairement), a favorisé, souvent à son insu, des attitudes fixistes et simplificatrices trop répandues dans le milieu des services techniques et que nous avons déjà évoquées plus haut («Il suffit d'isoler», « Il suffit de corriger l'éblouissement», etc.). En ce sens, les thématiques du milieu qui prennent mieux en compte la rétroaction du vivant sur son contexte se substitueraient avantageusement à celles de l'environnement plus enchaînées aux états physiques isolés posés comme premiers: stabilité du signal mesurable à partir duquel on analyse les réactions. Par exemple, la notion de «qualité environnementale» que nous souhaitons voir remplacer celle de nuisance dans les préoccupations sanitaires et politiques devrait elle-même être comprise plutôt comme un processus permanent de qualification dynamique produit par la concurrence entre les diverses composantes physiques et humaines. On peut d'ailleurs se demander si la ville qui est, par nature, le champ d'une compétition permanente entre une multiplicité de dynamiques ne représente pas l'environnement par excellence. N'est-elle pas le modèle le plus ancien, le plus archétypique et le plus accompli d'une maîtrise perpétuellement intégrative de l'espace, des contraintes physiques et biologiques, des acquis de la culture et des techniques humaines? Ville et environnement seraient alors deux faces de la même réalité en mouvement.

\section{Environnement sensible ef ambiances architecturales}

Le tableau que nous venons de brosser rapidement voulait montrer les conditions de possibilité à partir desquelles on peut comprendre l'urbain comme un environnement. Mais de quel environnement parlons-nous? On aura noté que sous l'angle interdisciplinaire et situé que nous avons développé, la notion paraît subir un élargissement peu conforme à la compréhension actuelle fortement marquée par les caractères majeurs du modèle physique que nous avons évoqués plus haut. Venant de la langue scientifique américaine récente, cet environment désigne strictement le faisceau des conditions physiques, biologiques et sociales agissant sur le vivant. Il serait inutile de remonter l'inéluctable dérive linguistique et de vouloir réhabiliter la définition antérieure qui, en français, depuis le XII e siècle, 
indiquait l'entour des phénomènes, la contextualité locale qui est au centre de nos préoccupations.

L'actuelle notion d'environnement affiche donc une configuration particulière qui, d'une part sélectionne dans sa compréhension les caractères assimilables par le modèle des sciences de la nature et, d'autre part, élargit son extension à tout phénomène de nuisance. En même temps, telle que médiatisée, la voici chargée d'une forte polysémie et d'une surdétermination où s'entremêlent confusément l'urgence, le sanitaire, le planétarisme, le militantisme. Nous noterons ainsi que la sensibilité écologiste militante, quelque déclaration d'intérêt pour le local qu'elle proclame, valorise trop souvent ce réductionnisme en hypostasiant les nuisances du moment et en négligeant presque toujours la production sociale des représentations qui les désignent comme telles. Les quelques récents travaux sur les conceptions de l'environnement dans les siècles passés montrent que l'émergence globale de telle nuisance plutôt que de telle autre n'est pas nécessairement fonction de la qualité physique ou technique du signal, mais bien des sensibilités de l'opinion, du type de relations sociales et des comportements de classe (19).

Telle que traitée par la recherche architecturale et urbaine, la notion d'environnement connaît donc un double écart (20). Plus large, sa compréhension est ouverte à plusieurs modèles d'intelligibilité. Plus restreinte, son extension s'applique aux phénomènes dont la

(19) Cf. A. Corbin, op. cit.; P. Goubert et al., Du Luxe au confort. Paris: Belin, 1988; O. Balay, Discours et savoir-faire sur l'aménagement de l'environnement sonore urbain au XIX ${ }^{e}$ siècle. Grenoble: CRESSON, thèse, 1992; G. Chelkoff et al., Bien-être sonore à domicile. Architectures du logement et potentiel de confort sonore. Grenoble: CRESSON, 1991.

(20) On trouvera utilisée dans les milieux de la recherche architecturale la notion d' "environnement construit». Un laboratoire helvétique l'a même intégrée dans son intitulé: «l'Institut de recherche sur l'environnement construit» (IREC/EPFL). Plus précise qu'«environnement», renvoyant clairement à l'édifié et à l'aménagé, on pourra en user comme d'un commode synonyme. Elle présente toutefois l'inconvénient d'induire une connotation prévalante liée aux sciences de la construction et, par ailleurs, de valoriser une acception factuelle aux dépens de la dimension opératoire. qualité est appréhendée par les sens avec une précision suffisante pour que trois opérations soient possibles: la perception effective des variations (dimension du vécu singulier), l'investissement par des représentations esthétiques et axiologiques (sens contextualisé du phénomène), l'intégration dans les processus d'instrumentation du lien social (sens et références collectives du phénomène) (21). On ajoutera à ces trois critères un quatrième qui nous concerne directement: les composantes de l'environnement retenues sont celles sur lesquelles l'architecture et les formes urbaines ont une répercussion structurelle; c'est-à-dire que leur perception inclut les modalités morphologiques du lieu et que ces composantes sont, de droit, partie intégrante du processus de conception et de toute projétation.

Deux objets notoires du savoir écologique restent donc périphériques pour l'instant: la pollution de l'eau et la pollution de l'air qui ne répondent pas à l'un ou l'autre de ces critères. Ainsi, la perception des qualités gustatives et olfactives de l'eau ou de l'air reste un indice culturellement peu développé et de faible prédictibilité pratique quant à la réalité chimique et à ses effets physiologiques. Ainsi, la pollution de l'eau peut induire des réactions revendicatrices collectives, elle n'est pas pour autant un support sensoriel des échanges interpersonnels. Ainsi, l'organisation du milieu urbain a évidemment des effets sur ces pollutions, mais la principale maîtrise du phénomène n'est pas du ressort de l'architecte ou de l'urbaniste.

Quelles nouvelles désignations évoqueront ces notables différences? Nous en proposons deux: environnement urbain sensible et ambiances architecturales et urbaines. En parlant d'environnement sensible nous développons et diversifions une région, la «dimension humaine» qui, dans le champ couvert par le concept général (environnement) est essentiellement référée au schéma behavioriste.

(21) Ces trois critères sont à comprendre comme des potentialités effectives. Les sensata ne sont ni nécessairement, ni toujours accomplies en perceptions conscientes, utilisées pour faire du lien social, affectées de jugement. Mais il est nécessaire qu'elles puissent l'être selon les occasions et les règles de la vie quotidienne et que cette effectivité soit directement ou indirectement observable. 
En utilisant l'adjectif sensible nous renvoyons plus largement au champ global des perceptions et des usages localisés et datés, étant entendu que la dimension configuratrice et active affecte l'intégralité de la construction perceptive: données proprement physiques, facteurs individuels, facteurs culturels et sociaux. Conforme à l'approche in situ de l'environnement urbain que nous avons décrit auparavant, cette acception ne présente qu'un défaut: elle s'applique mieux à l'analyse interdisciplinaire des situations quotidiennes données, à la ville naturée, qu'à l'articulation entre théorie et pratique architecturale.

Une autre notion conviendra mieux à la ville naturante, à l'espace urbain en tant que production: c'est le terme pluriel d'ambiances architecturales ou d'ambiances urbaines. Les ambiances lumineuses, thermiques, sonores sont aussi bien des phénomènes sensibles que des phénomènes produits. Dans le champ professionnel et pédagogique des sciences appliquées à l'architecture, la notion est d'ailleurs associée fortement à celle de maîtrise («maîtrise des ambiances»). La compréhension d'une ambiance repose en fait sur trois composantes: la part des sciences et techniques, la part du savoir sur les usages et représentations quotidiens, enfin, la part de l'art.

Si le premier champ suit les progrès de l'instrumentation et de la modélisation issus de la physique appliquée, si le second évolue avec les nouvelles attitudes des sciences de l'homme et de la société devant l'observable, le troisième champ est-il la boîte noire? Quelle est cette part de l'art?

La production des ambiances appelle art et savoir-faire à double titre: à titre trop souvent implicite dans tout acte architectural ordinaire, à titre de compétence experte dans l'actuel développement des aspects sensibles de l'aménagement urbain. Dans la pratique du projet d'architecture ou d'urbanisme, tout plan de masse, toute composition formelle, tout choix de matériau entraîne nécessairement des effets lumineux, sonores, thermiques, tactiles dont la portée d'ensemble n'est ordinairement maîtrisée que dans la limite des obligations techniques réglementaires. Le reste, c'est-àdire ce qu'on ne peut assimiler à une nuisance est presque toujours ignoré, ou jugé insignifiant, ou recouvert par un jugement esthétique portant sur les seules apparences visibles. Le reste serait un domaine inconnu, imprédictible, bienfaits et méfaits d'ambiances ne devant apparaître qu'après l'édification (22).

Ainsi, en conséquence de cette conduite magique, mais aussi par nécessité de gestion du bâti, il existe un second champ de production des ambiances. Présente, la part de l'art y est inégalement partagée. Dans la pratique la plus courante, les ambiances urbaines sont traitées et gérées par des intervenants fort divers et plus reproducteurs de savoirfaire que véritables créateurs: ainsi l'entreprise qui appose une isolation extérieure sur des immeubles anciens, ou le technicien municipal qui implante des lampadaires, ou l'électronicien qui installe une sono temporaire pour une braderie ou, encore, une régie de transport en commun qui tolère des musiciens ambulants. Bien différente est la compétence artistique chez les nouveaux auteurs de ce que l'on peut appeler scénographie urbaine. Les actions de mise en scène lumineuse et sonore de la ville furent d'abord l'apanage des artistes du land art comme Christo ou Max Neuhaus. Aujourd'hui, elles s'intègrent lentement dans l'action globale d'aménagement urbain où elles apportent la part de l'imaginaire et du spectaculaire (23). Ainsi, en France, Nicolas Frize transforme la pratique du compositeur en résidence en travaillant directement sur l'espace et avec la population de la ville qui l'accueille. Ainsi, Laurent Fachard en transposant l'esthétique de son maître en éclairage de scène, Henri Alekan, au traitement des façades, des monuments, produit une épiphanie lumineuse de la ville (24).

(22) Cette attitude, n'est bien évidemment qu'un mécanisme de défense, aucune des justifications ordinairement alléguées, y compris celle touchant à l'économie du projet, ne résiste à un examen attentif. La carence de formation et de diffusion des connaissances sur ce sujet a conduit plusieurs laboratoires de recherche (CERMA, CRESSON, ISITEM, LASH, CSTB) à créer un DEA «Ambiances architecturales et urbaines».

(23) Les cas sont encore rares mais spectaculaires où une ville planifie un urbanisme lumineux, par exemple. Le «Plan lumière» de la ville de Lyon mis en cuvre depuis cinq ans joint ainsi l'agréable au fonctionnel et au sécuritaire.

(24) Sur ce réemploi urbain des techniques et savoir-faire des arts du spectacle et les premières analyses sur ce parallèle entre la place et la scène, on se référera au séminaire Scénographie et espaces publics organisé à l'Arche de la Défense par le Plan Urbain (ministère de l'Équipement) en 1993 et dont les Actes devraient paraître bientôt. 
La notion d'ambiances architecturales intègre donc plus facilement cette part de l'art, du savoir produire l'espace urbain qui reste étrangère au champ sémantique de l'environnement. En revanche, le pluriel de la notion - ambiances - trahit un handicap non négligeable. En l'état actuel des connaissances, parler d'ambiance urbaine, au singulier, ne correspond à rien de précisément analysable, si ce n'est dans la succession des registres sensoriels, chaque sens impliquant des logiques d'observation et des outils spécifiques. Et aucune confrontation interdisciplinaire ne pourrait être faite. Nous avons des connaissances sur l'ambiance thermique, l'ambiance sonore, l'ambiance lumineuse, voire sur l'ambiance olfactive à laquelle on commence à s'intéresser mais nous manquons actuellement d'une théorie transsensorielle couvrant également les différentes disciplines concernées par la perception et la variété des sensations. Ne peuvent en tenir lieu ni certains travaux sur les interactions binaires conduits en physiologie et en ergonomie, ni la célèbre et hypothétique théorie des correspondances, ni même l'existence de modèles explicatifs généraux que l'on suppose applicables à tous les sens ou presque tous (25).

À l'issue d'une longue confrontation avec la recherche environnementale, la recherche architecturale et urbaine aboutit donc à produire deux notions sans doute plus précises que celle d'environnement. L'une - environnement sensible - répond mieux aux démarches de recherche visant le donné urbain, l'autre - ambiances architecturales - convient mieux aux investigations visant la connaissance de la production de l'espace construit. La prédilection pour l'une ou l'autre des notions est commandée par les objectifs momentanés de recherche ou d'enseignement mais elle ne prend sens que sur la base d'une nécessaire complémentarité. Si certains travaux sur la ville restent confinés dans une répétition compulsionnelle de la thématique des nuisances, si le thème pédagogique de la maîtrise des ambiances en architecture est resté longtemps et trop souvent une stricte affaire d'application mécanique de normes et de recettes techniques, c'est précisément par défaut de

(25) Ainsi, des concepts clés de la Gestalttheorie comme ceux de figure et fond restent bien plus pertinents pour la vision que pour l'audition. circulation entre la connaissance interdisciplinaire de l'environnement sensible et la réflexion théorique et méthodologique sur les ambiances.

\section{Apports des travaux sur l'environnement sensible et les ambiances à la recherche architecturale et urbaine}

Si la recherche sur la ville abordée comme un milieu sensible a diversifié et fécondé une partie du vaste champ des sciences de l'environnement, l'inverse est vrai. Qu'est-ce que les travaux sur l'environnement sensible et les ambiances apportent de nouveau à la recherche sur l'architecture et la ville?

L'une des principales difficultés de la recherche en urbanisme et surtout en architecture fut et reste l'articulation entre la connaissance de l'architecture archilecturée el celie de l'architecture architecturante. Entre d'une part, l'analyse de l'architecture comme objet offert à l'histoire, à la sémiologie, à la psychologie, à la sociologie, à l'épistémologie, à l'esthétique, à la physique appliquée et, d'autre part, l'analyse des processus de production de l'espace bâti. Parmi les diverses raisons d'un blocage qu'il serait trop long d'analyser ici, il faut retenir la concurrence des apanages. D'un côté, la part du savoir, de la spéculation, de l'autre, la part de l'art et des savoir-faire. Tout entretenu qu'il soit par les crispations polémiques, ce dualisme ne laisse pas de faire symptôme. C'est bien la nature profonde du projet qui est en question. Comme si l'architecture ne pouvait accéder au réel qu'à partir d'une rupture, d'un saut qualitatif du connu à l'inconnu.

Or, il semble que l'articulation, plus haut évoquée, entre les deux approches complémentaires de l'environnement construit apporte quelques indications sur la possibilité de rétablir un dialogue entre l'art et la technique, le talent et les compétences, le savoir et le savoir-faire. En se limitant à un aspect bien circonscrit du phénomène urbain, en pratiquant une interdisciplinarité large pratiquée par une communauté de chercheurs aussi réduite qu'ouverte, le champ étant nouveau, la recherche sur l'environnement sensible et les ambiances architecturales semble offrir quelques moyens pour changer les façons de voir, tourner certains blocages, produire des notions et des méthodes transverses et utiles, en pratique comme en théorie. 


\section{Sur les autres sens de l'architecture}

Depuis la Renaissance, à l'instar des autres savoirs raisonnés, l'architecture est sous l'emprise majeure du visuel (26). L'œil est devenu le sens des sens, non seulement pour fonder le dessein dans le dessin, mais aussi pour connoter fondamentalement le langage de l'analyse et diriger la rhétorique de la conception architecturale (27). Voir va de soi. Du plan à la maquette, du dessin au chantier, la production des formes édifiées dépend tellement de la vision qu'on oublie même cette hégémonie.

En revenant à l'état premier des choses, dans l'ordre de la perception, la recherche sur les ambiances architecturales rencontre une collection de signaux et de sensata égaux en droit. S'interroger sur la nature d'une forme non visible, suivre la logique de l'audition ou de l'olfaction pour décrire un territoire habité, se demander à quoi ressemble une composition paysagère thermique, olfactive ou aérodynamique: rien de plus scientifiquement légitime (28). Mais rien de plus inquiétant pour les certitudes de la pensée architecturale et urbaine. Car il n'est pas de nécessaire orthogonalité dans un espace sonore, un espace olfactif, un espace thermique. Ni de congruence obligée entre les limites visuelles, sonores, tactiles qui sont d'une nature bien différente. La référence spatiale, telle que définie par

(26) Faut-il préciser que si le panoptisme, au sens épistếmologique, trouve à la Renaissance les conditions d'une propagation rapide et généralisée dans les arts, les techniques et les lettres, comme l'ont montré des auteurs aussi différents que Rudolf Arneihm, Eugenio Garin, Ervin Panofsky, Ernst Gombrich, Marshall MacLuhan, Michael Baxandall, la prédominance culturelle du visuel en Occident trouve ses racines dès l'Antiquité méditerranéenne. L'histoire de cette construction culturelle remarquable fait l'objet actuellement de différents travaux, en particulier sur le thème de la vision paysagère.

(27) Sur cette logique pratique de la conception urbanistique et architecturale qui ressortit à une rhétorique, on pourra lire nos analyses et le tableau comparatif en annexe, in Pas à pas. Paris: Le Seuil, 1979.

(28) Cette démarche engagée suppose un immanentisme sensoriel de la pensée (cf. nos récents développements sur cette hypothèse: J.-F. Augoyard, "La sonorización antropológica del lugar", in M.J. Amerlinck dir., Hacia una antropología arquitectonica. Jalisco (Mexico): Universidad de Guadalajara, Colección «Jornadas Académicas», 1995). les caractères newtoniens, perd sa pertinence explicative dans les processus sensibles autres que le voir. Plus encore, on peut montrer que le visible n'est pas entièrement soumis à la spatialité cartésienne (29). Pourtant, comme il est difficile d'imaginer des représentations du tactile, du thermique, de l'olfactif, qui obéiraient à la logique propre à chacune de ces sensations! Comment faire admettre à l'architecte qu'une forme sonore, c'est d'abord du temps? «Et comment la dessinerais-je?», dira-t-il.

À ces résistances, on voit combien la métaphore visuelle a pénétré en profondeur notre manière de penser l'espace construit (30). Est-il illusoire de vouloir rétablir cet équilibre des sens que l'architecture vernaculaire, ou le bâtir intuitif non soumis à l'hégémonie visuelle paraissent avoir mieux respecté (31)? Il est au moins nécessaire d'inquiéter les attitudes mentales des architectes et urbanistes trop oublieux de l'existence des autres sensorialités. La réhabilitation des sens devenus mineurs passe par trois tâches. La première porte sur la critique des stéréotypes présidant à la programmation. La seconde doit œuvrer à modifier l'attitude cognitive sur l'espace construit. La troisième intervient sur l'imagination créatrice architecturale.

Dans la pratique constructive, il faut démontrer, études à l'appui, la possibilité et le gain formel, social et économique d'une intégration préventive de tous les éléments de confort, dès le stade de la programmation. Le coût économique et social des malfaçons gui causent dommage à la santé ou au

(29) Pour de plus amples développements, on pourra se référer à J.-F. Augoyard, "La vue est-elle souveraine dans l'esthétique paysagère?», Le Débat, n 65, mai-août 1991, Paris, Gallimard, p. 51-59.

(30) Des travaux comme ceux de Grégoire Chelkoff montrent non seulement ce qu'il advient d'un espace public analysé par l'écoute, mais que l'espace public peut aussi être vu comme une configuration qui échappe au modèle d'espace newtonien. G. Chelkoff, Entendre les espaces publics. Grenoble: CRESSON, 1988. G. Chelkoff, J.-P. Thibaud, Les Mises en vue de l'espace public. Grenoble: CRESSON, 1992.

(31) C'était l'une des questions posées au colloque Au-delà du paysage moderne organisé par l'Association pour la recherche à l'École des hautes études en sciences sociales, sous la direction d'Augustin Berque, Paris, Centre Pompidou, 19-20 octobre 1990. Cf. compte rendu des communications dans Le Débat, n 65 , op. cit. 
bien-être des usagers dépasse toujours le prévisible et l'éventuel surcoût des procédures constructives. L'argument souvent allégué par les responsables des projets architecturaux et urbains pour ne pas dépasser les limites inférieures de confort imposées par les réglementations s'appuie sur une logique du surajout, ou de surdimensionnement du matériau. On ne pense pas à l'efficacité d'une astucieuse organisation de l'espace et d'un dessin des formes qui seraient favorables par eux-mêmes à tel effet acoustique ou thermique.

Comment rendre possible cet exercice? C'est la tâche de l'information et, plus encore, de la formation. Travail de longue haleine, car il s'agit bien de modifier une attitude cognitive forgée depuis la Renaissance et qui imprègne non seulement les études d'architecture mais l'ensemble du cursus éducatif (32). Par exemple, à rester dans une modalité strictement théorique, l'apprentissage des propriétés de l'environnement sonore est de faible intérêt. Une véritable compréhension de ce qu'il faut appeler une audition du monde - au sens d'une Weltanhörung — et la fixation des connaissances en la matière passent nécessairement par une pédagogie de l'écoute, par un itinéraire de réforme des habitudes auditives acquises, lesquelles sont, la plupart du temps, des attitudes de non écoute. Sans cette propédeutique, on ne peut espérer que l'architecte, au moment où la morphologie de l'espace constructible apparaît, ait la capacité de penser forme sonore au même titre que forme visible.

Sans cette expérience sensible incorporée qu'il faudrait appliquer à chacun des canaux sensoriels, les qualités sonores, aérodynamiques, olfactives, tactiles de l'espace ne seront jamais que des adjonctions à la visibilité de la forme créée; et l'empreinte des sens (33) oubliés ne pourra jamais

(32) On peut aisément s'en convaincre en faisant l'analyse structurale des modalités langagières utilisées dans les injonctions et consignes didactiques dès les premières années de l'école. La connotation visuelle prédomine dans les verbes, dans les notions, dans les adjectifs descriptifs y compris quand il s'agit d'écoute, d'olfaction ou de tact.

(33) L'Empreinte des sens, c'est le titre d'un récent bilan des connaissances actuelles à l'interface de la physiologie et de la psychologie. L'auteur Roger Ninio y développe précisément la prégnance de la «raison perceptive» comme un thème majeur. R. Ninio, 1989, L'Empreinte des sens. Paris: Odile Jacob/Le Seuil. être prégnante, ni participer avec efficacité à la production de la forme. La troisième tâche de la recherche, c'est de faciliter l'exercice ordinaire d'un imaginaire bâtisseur aussi inventif pour l'écoute, la motricité, l'olfaction, la relation avec l'air, que pour le voir. Cette qualification polysensorielle délibérée et souhaitable pour tout genre d'architecture ne dérivera pas simplement des savoir-faire spécialisés utilisés dans les architectures d'exception: opéras, salles d'écoute, bâtiments d'exposition, maisons solaires expérimentales. La démarche constructive de l'architecture ordinaire doit inventer, selon sa propre économie, des processus et dispositifs de confort idoines (34). Une enquête ethno-historique reste à faire sur les savoirs et savoir-faire intuitifs et inventifs suscités par les nécessités naturelles mais aussi culturelles de maîtriser les éléments de confort dans l'architecture ordinaire (35).

\section{Sur l'interdisciplinarité architecturale}

En tant que savoir cumulatif, le champ de l'archilecture est pluridisciplinaire par nature, que les diverses connaissances cultivées coexistent simplement, ou qu'elles soient confrontées, ajustées sur un fond épistémique commun, ce qui est plus rare. Pourtant le travail de conception à l'œuvre dans tout projet d'architecture engage une véritable interdisciplinarité, c'est-à-dire une confrontation concurrentielle et dialectique entre des savoirs et des processus de nature différente.

Qu'en dit le praticien? Que le projet abouti est une affaire d'expérience, de savoir-faire, de négociation entre des contraintes, avec une part aléatoire, événementielle dépendante de l'histoire du projet. La mise à jour du fond épistémique de l'architecture est déjà ancienne. Elle s'éclaire en partie et progressivement depuis le romantisme, chaque fois qu'un auteur aborde la question de l'essence de cet «art» atypique qui mêle fonction et beauté (36). Le fonctionnement

(34) On pourra examiner en ce sens l'intéressante expérience des «Maisons des cinq sens» édifiées par L'Effort Rémois, voici quelques années.

(35) C'est l'un des thèmes majeurs du réseau international: La Qualité sonore des espaces habités, actuellement géré par le CRESSON.

(36) Faut-il rappeler que cette question de savoir comment l'architecture peut prendre place parmi les arts court de Hegel et Schopenhauer à Christian Norberg-Schultz, en passant par Paul Valéry, le Bauhaus, Le Corbusier? 
cognitif a été beaucoup moins creusé. Quelle est la nature de cette mystérieuse unité qui lie dans l'acte architectural des connaissances très différentes et qui, par ailleurs, articule des savoirs et des savoir-faire? Même si l'architecturologie en est à ses débuts, on doit savoir gré à Philippe Boudon d'avoir délibérément centré la question dans le champ de l'épistémologie.

Parmi les questions sur l'architecture ainsi reformulées, l'une des plus importantes est de savoir par quels processus le fond épistémique peut articuler la collection des savoirs concernant l'architecture. Or, la question écologique qui oblige à traiter les relations entre la dimension humaine et la dimension physique des phénomènes situés s'apparente, sur ce point, à la question architecturale. On comprend dès lors que l'entreprise descriptive et taxinomique appliquée aux formes architecturales sous les espèces de la typomorphologie est loin de pouvoir fonder à elle seule le savoir architecturologique. Comme la mémoire, l'architecture n'est pas qu'un polypier d'images. Il suffit de quitter le champ du visible pour que les types et les classes perdent leur pertinence (37).

L'actuel développement des recherches françaises sur les ambiances architecturales tend à montrer que la morphologie architecturale est fondée sur les interdépendances structurelles entre forme construite, forme perçue, forme représentée. Impossible d'expliquer le fond d'une de ces modalités sans se référer aux autres. Les trois opérateurs de cette conjonction modale n'ont rien de mystérieux, ce sont le signal physique perceptible, l'ensemoje des normes, règies et codes, enfin les instrumentations, fonctions et usages affectés

(37) Une intéressante tentative fut faite en 1980 par un acousticien et un chercheur architecte pour tester dans le champ du sonore la pertinence typomorphologique. Or, l'application des types et des rapports d'échelle n'est valide que pour un habitat à forme fermée (type îlot avec cour intérieure) et que pour ce qui touche au coefficient d'isolation. Pour le reste, les phénomènes sonores ne correspondent pas aux découpages et emboîtements classificatoires proposés par la typomorphologie architecturale. Cf. P. Panerai, D. Tatutesco, Diversité sonore et formes urbaines. Paris: ADROS/UP3, 1983, 2 tomes. Sur cette analyse critique, cf. J.-F. Augoyard et al., Sonorité, Sociabilité, Urbanité. Méthode pour l'établissement d'un répertoire des effets sonores en milieu urbain. Grenoble: CRESSON, 1982, 155 p. +2 cassettes (120 minutes). à la forme construite. Les travaux actuellement attachés à étudier le fonctionnement complexe et interdépendant de ces opérateurs devraient aider à mieux comprendre non seulement la nature de l'environnement sensible, mais plus largement les processus interdisciplinaires impliqués dans l'acte de production de l'espace construit.

\section{Sur la nécessaire connaissance de l'usage}

La connaissance des pratiques et usages de l'espace habité est un thème majeur de la recherche urbaine et architecturale depuis trois décennies. L'influence méthodologique de ces travaux sur l'observation de l'environnement sensible est importante, comme nous l'avons indiqué plus haut. En retour, les nouvelles connaissances sur les ambiances architecturales devraient apporter leur pierre à l'édification de la connaissance de l'usage de l'architecture. Ceci de deux façons: d'une part en restaurant la fonction des facteurs physiques dans la perception de l'espace, d'autre part en mettant en valeur l'instrumentation des constructions perceptives et du lien social in situ.

Rappelons que la question de la perception de l'espace bâti a connu trois traitements bien différents dans la recherche française de ce demi-siècle. Soit par le développement d'une psychologie expérimentale souvent soucieuse de dépasser ses origines behavioristes en cherchant des ouvertures du côté de la psychosociologie compréhensive (38). Soit par le développement de la veine phénoménologique qui a été cultivée avec un bonheur très inégal par les chercheurs en architecture ou en urbanisme, tant l'usage de l'organum logique et verbal du champ posthusserlien, si séduisant soit-il, ne peut dispenser du patient labeur des réductions, de cette attention méticuleuse accordée aux choses sans laquelle l'entreprise phénoménale tourne à vide (39). Soit enfin, à travers certains travaux de sociologie urbaine traitant des représentations collectives d'objets comme le paysage, l'habitat, l'espace public, le patrimoine.

(38) Exemplaire était en ce sens la dernière synthèse offerte par la regrettée Elizabeth Dumaurier: Psychologie expérimentale de la perception. Paris: PUF, 1992.

(39) Il est remarquable que les plus ambitieuses tentatives d'analyse phénoménologique de la perception de l'espace construit aient été menées dans les années 1960 et 1970 par des 
La recherche sur les ambiances n'a pas l'ambition de réconcilier ces courants différents où la force des méthodes et l'intérêt des résultats tiennent, en partie, de la spécificité du point de vue choisi, du parti antagonique délibéré. Mais, nous l'avons souligné, l'observateur d'un phénomène d'environnement in situ ne peut faire abstraction ni du signal physique, ni du traitement imposé par la perception, ni de la composante collective. Autrement dit, il affronte nécessairement des articulations problématiques dont les autres courants de recherche ont fait l'économie.

Ainsi, en s'élevant contre le substantialisme physique entraîné par un behaviorisme réducteur qui n'accordait aucune valeur causale au collectif, la sociologie urbaine a rétabli, avec raison, l'autonomie des causalités sociales. La contrepartie en est la méfiance tenace pour tout ce qui touche à la matérialité de l'espace. Or, c'est oublier que depuis vingt ans, les sciences du comportement ont évolué. N'entend-on pas dire de plus en plus souvent, en physiologie de la perception, que l'environnement physique n'est ni plus ni moins qu'une liste de propriétés offertes à une infinité de constructions perceptives?

Ainsi encore, le développement de la raison sociologique, au cœur du phénomène urbain, pourrait tendre à produire un idéalisme du lien social. Comme si l'ordre de l'analyse finissait par créer les conditions d'existence du quotidien, comme si le savoir, oublieux des sens, produisait une réalité privée de sensation. Pourtant, les interactions sociales n'auraient aucun efficace sans l'instrumentation sensorimotrice qui les incorpore. Comment penser, dès lors que les marques sensibles n'auront aucun effet sur le message sinon au titre d'un hylémorphisme qu'on ne peut plus soutenir aujourd'hui et que l'observation élémentaire dément (40)?

auteurs dont l'inspiration théorique est pluraliste. Ainsi, Henri Lefebvre jamais totalement oublieux de l'influence marxiste, Abraham Moles toujours très au fait de la psychologie expérimentale d'où il tire certains modèles explicatifs. Quant aux phénoménologues urbains véritablement attentifs aux choses et aux gens, il faut en reconnaître la rareté. Peu d'entreprises peuvent rivaliser sur ce point avec celle de Pierre Sansot.

(40) C'est, par exemple, au nom de cette immanence du sensible dans les interactions sociales que la figure de l'étranger est déclinée avec des significations et des valeurs bien différentes selon le marqueur sensoriel: vue, audition, olfaction, tact...
La recherche sur l'environnement sensible aborde donc le signal physique non seulement comme matière à perception de l'espace mais aussi comme instrument du lien social dans l'espace. Elle développe ce thème en deux sens complémentaires. La question: «comment du social peut-il être du sensible?» est l'objet de la praxéologie de la perception et de l'observation, très peu menée jusque-là, de l'instrumentation des relations sociales (41). La question: «comment du sensible peut-il être du social?» est l'objet du développement de l'esthétique - entendue comme théorie de l'organisation des sensations - en deux directions jusque-là peu abordées: l'expérience esthétique comme action et comme savoir-faire partagé.

L'architecture et la ville ne sont pas que des objets. Leurs effets sensibles agissent au centre d'un nœud rassemblant perception et action, forme et fonction, individu et collectivité. Dès lors, l'architecte et l'urbaniste peuvent-ils penser à l'usage de l'espace construit sans savoir comment l'usage les reconstruit? On peut présumer que, avec l'avancement des travaux sur l'environnement sensible, la compréhension du rapport entre l'usager et le professionnel ou l'expert de l'espace connaîtra quelque évolution. L'environnement sensible de l'usager ne serait plus seulement envisagé comme un objet de consommation, voire de contemplation, et d'action au sens revendicatif. La connaissance des modalités de la pratique quotidienne devient prioritaire. Et comment se contenter alors des outils conceptuels et des modèles explicatifs reçus? La mise à jour des interdépendances (42) exige des outils de nature paradigmatique comme l'effet, le motif, l'ambiant, le transcript (43) et des procédures d'élucidation virtuelle comme la modélisation numérique.

(41) C'est l'objet essentiel des travaux de Jean-Paul Thibaud depuis quelques années: J.-P. Thibaud, J.-P. Odion, À l'écoute du chantier. Des productions sonores aux modes de prévention. Paris: Plan-Construction, 1990; J.-P. Thibaud, Le Baladeur dans l'espace public urbain. Essai sur l'interaction sensorielle de l' interaction sociale, thèse, université de Grenoble 2, 1992.

(42) Cf. infra: Les Raisons écologiques de l'urbain.

(43) Ce travail d'élaboration de paradigmes est mené particulièrement dans le cadre de la collaboration entre le CRESSON et le CERMA (n.cit.). La notion de transcript est proposée par l'architecte Bernard Tschumi. 


\section{Sur la réhabilitation de la connaissance esthétique comme savoir interdisciplinaire}

Objet de très nombreux travaux en histoire de l'art, l'architecture a mérité assez peu de discours esthétique au sens baumgartien du terme: théorie du fondement du beau par une sensibilité autonome (44). Il semble plus facile de traiter de la production des formes bâties, de classer les genres, les styles et les écoles, que d'analyser la réception de l'œuvre architecturale ou urbaine. Les brèves et partiales pages de Hegel dans son Esthétique ressortissent plutôt à une théorie de la hiérarchie symbolique des formes, du fait de leur caractère prescriptif. Peu d'auteurs se sont risqués sur le sujet de la perception de l'architecture. Ils l'ont fait dans la perspective d'une critique des a priori du jugement de goût mais à toutes fins de réformer les représentations de la ville (Camillo Sitte) ou des monuments (Aloïs Riegl). On trouvera chez des psychologues et des philosophes de l'art comme Ervin Panofsky, Pierre Francastel, sir Ernst Gombrich ou encore Nelson Goodman, des remarques éclairantes sur le lien entre l'organisation perceptive et les structures de la représentation de l'œuvre architecturale. De même on trouvera, sur le sens de l'architecture, des théories aussi célèbres que celle de Venturi ou celle de Christian Norberg-Schultz dont la veine phénoménologique nous rapproche du sentir. Pourtant l'approche des modalités sensibles de la perception d'une forme construite n'est guère plus abordée par les approches plus psychosociologiques qui s'appuient avec prudence sur un socle d'interprétation sémiologique des représentations de l'usager plutôt que sur l'observation directe de l'expérience sensible (45). Entre

(44) Ne sauraient en tenir lieu nombre de publications qui sous le nom d'«esthétique de l'architecture» décrivent les caractères formels d'architectures données, à tort ou à raison, comme œuvres à classer. C'est de nouveau la question du style qui prédomine, non celle de l'expérience esthétique de l'usager. On peut mettre dans la même catégorie les argumentaires de programmes architecturaux qui décrètent de façon apodictique la rusticité, la modernité, la transparence, ou encore l'urbanité de bâtiments dont on se garde bien d'évoquer les effets sensibles prévisibles dans l'usage quotidien.

(45) Cf. la sémiotique des styles vernaculaires chez Sylvia Ostrovesky (L'Imaginaire bâtisseur. Paris: Librairie des Méridiens, 1983) et la notion de «compétence esthétique ordinaire» chez Marion Segaud (Thèse, université de Nanterre, 1989). l'expérience esthétique de l'œuvre d'architecture et l'expérience sensible du bâti, le hiatus est béant.

À quoi tient cette difficulté? D'abord au fait que l'architecture déborde le domaine de l'art proprement dit en deux façons puisque, pour conserver la définition triadique de Vitruve, elle ajoute à la beauté (venustas), seul objet de contemplation artistique, la technicité constructive (firmitas) et la fonctionnalité d'usage (commoditas). Il est donc peu probable que l'esthétique architecturale connaisse des progrès tant que deux obstacles ne seront pas levés. D'une part, quel rapport entre la création formelle et la technique? D'autre part, quel rapport entre la forme bâtie et la forme habitée?

L'habitant étant à la fois usager de fonctions et pratiquant de formes sensibles, l'environnement construit croise de façon aiguë deux questions qui sont, en fait, au cœur du débat esthétique contemporain sous la forme suivante: savoir où passent les frontières de l'artistique, savoir quel est le rôle du spectateur. Pour élucider l'articulation paradoxale entre forme et fonction, les outils sont encore rares dans le champ de l'esthétique générale. Une filière née de l'esthétique allemande du XIX ${ }^{e}$ siècle, continuée par Wörringer, puis par le Bauhaus et ceux qui s'en inspirent, donne un ensemble d'hypothèses qui attendent de plus amples développements. Quant au rôle du spectateur, on découvre que le thème, avant d'avoir été approprié (et souvent réduit) par la sociologie de l'art au cours de ce siècle, a fait l'objet, dès le XIX ${ }^{\mathrm{e}}$ siècle, d'observations fondamentales au niveau de la construction sensible et culturelle. Ainsi naît une sorte de pragmatique du spectateur, plus près de nous reprise par l'École de Prague, l'École de Constance et l'Institut Warburg (46).

Or, nous trouvons un point commun capital entre ces deux thématiques apparemment différentes. Les analyses convergent toutes sur des questions touchant à l'organisation perceptive contextualisée. Quelles sont les logiques propres à chaque sens? Comment est articulée l'intersensorialité par laquelle nous accédons à l'unité de la forme? Comment

(46) Les traductions françaises nous proposent en particulier: E. Gombrich, L'Art et l' illusion. Paris: Gallimard, 1986 (III. «Le rôle du spectateur»), H.R. Jauss, Pour une esthétique de la réception. Paris: Gallimard, 1990. 
l'usage orienté des sens (facteurs sociaux et culturels) intervient-t-il dans la construction du sensible (et non seulement du représenté)? Et ces questions sont elles-mêmes débattues sur le fond d'une hypothèse commune: notre relation avec l'environnement sensible et formel est à concevoir comme un échange, une circulation constructive entre le donné et le configuré, le senti et l'agi, le perceptible et le représentable.

C'est dans cette direction proprement écologique (47) que les travaux sur les ambiances architecturales trouvent des appuis théoriques et devraient pouvoir féconder une esthétique architecturale élémentaire prenant les phénomènes à la racine, c'est-à-dire selon l'itinéraire dont nous avons

(47) Le terme d'esthétique se réfère alors plus précisément au sens donné par Kant au début de la Critique de la raison pure. Il s'agit d'une esthétique élémentaire concernant toute organisation sensible. Le rapprochement de cette esthétique avec l'écologie entendue comme genre de savoir a déjà été fait par plusieurs auteurs (cf. en particulier Ernst Gombrich, L'Écologie des images. Paris: Flammarion, 1982, et le commentaire qu'il en fait dans la préface). esquissé la direction dans les paragraphes précédents. La forme construite est alors à considérer à quatre titres. Elle est un faisceau de propriétés physiques distinguées selon la nature de chaque genre de signal. Elle est une configuration sensible située. Elle est l'expression d'une culture d'experts alliant fonction, art et technique. Elle est l'expression-réception d'une culture d'habitants-usagers. On voit que l'étude de chacune de ces modalités renvoie à des champs de compétence déjà constitués. L'intérêt d'un programme développé au sein de la recherche architecturale tient justement à une approche transversale de ces attributs de la forme bâtie.

En pratiquant l'interdisciplinarité large que l'étude des formes in situ exige, l'esthétique architecturale peut quitter les rivages confinés de la taxinomie morphologique et stylistique. En devenant un lieu d'élection de la question du sens des sens, elle peut accéder au rang de connaissance fondamentale dans la recherche architecturale et urbaine. En cumulant la variété des savoirs sectoriels utilisés dans l'observation de l'environnement sensible et en les centrant sur la question de la forme, elle contribue à préciser le sens de la question sur la pratique devenue essentielle pour l'architecture d'aujourd'hui: quel est exactement le processus par lequel des formes construites adviennent à la réalité?

Vient de paraître dans la collection «Alidade»

\section{Stratégies spatiales : comprendre et maîtriser l'espace}

\section{A.S. Bailly, B. Guesnier, J.H.P. Paelinck, A. Sallez}

Quatre auteurs, issus de disciplines et de pays différents, ayant en commun leur souhait de faire connaître la science régionale, se sont réunis pour présenter, de manière simple, les principaux mécanismes, théories et modèles de la science régionale et leurs applications dans l'espace.

Cet ouvrage s'articule en quatre chapitres: • L'analyse spatiale : vers une conceptualisation; • Comprendre et maitriser l'évolution urbaine; $\bullet$ L'aménagement du territoire et la science régionale; • Politique, planification et stratégie régionale.

Par sa présentation simple, illustrée, il s'adresse avant tout au grand public, aux étudiants et aux personnes qui souhaitent s'initier à l'analyse spatiale.

1996,16 x 23, 216 p., 32 fig., biblio., index. ISBN 2-86912-058-6; prix: $100 \mathrm{~F}$

Diffusion:

La Documentation française, 124 rue Henri Barbusse, 93308 Aubervilliers cedex. Tél. (1) 483956 00, Fax (1) 48395601 